\title{
Compression Picks Item Sets That Matter
}

\author{
Matthijs van Leeuwen, Jilles Vreeken, and Arno Siebes \\ Department of Computer Science \\ Universiteit Utrecht \\ \{mleeuwen, jillesv, arno\}@cs.uu.nl
}

\begin{abstract}
Finding a comprehensive set of patterns that truly captures the characteristics of a database is a complicated matter. Frequent item set mining attempts this, but low support levels often result in exorbitant amounts of item sets. Recently we showed that by using MDL we are able to select a small number of item sets that compress the data well [11]. Here we show that this small set is a good approximation of the underlying data distribution. Using the small set in a MDL-based classifier leads to performance on par with wellknown rule-induction and association-rule based methods. Advantages are that no parameters need to be set manually and only very few item sets are used. The classification scores indicate that selecting item sets through compression is an elegant way of mining interesting patterns that can subsequently find use in many applications.
\end{abstract}

Keywords: frequent item sets, MDL, classification.

\section{Introduction}

Ever since the first paper on association rule mining [1], mining for frequent item sets has been a popular topic, as it has many useful applications. By now there are many algorithms that discover the frequent item sets efficiently $[1,5]$.

Another problem, however, is far from solved: the explosion of the number of results. Over the years, many solutions have been proposed, e.g., closed [15] and maximal [2] item sets. Most, if not all of these methods can be understood as a compression of the result set, some methods are lossless (closed item sets) while others are lossy (maximal item sets).

Recently, we proposed a radically different solution to this problem [11]. A set of item sets is interesting iff it yields a good (lossless) compression of the database rather than a good compression of the set of all frequent item sets. To determine whether or not a subset of the set of frequent item sets yields a good compression of the database, we used the Minimum Description Length Principle (MDL) [6].

As shown in [11], heuristic algorithms yield sets of frequent item sets that are easily four orders of magnitude smaller than the complete set of frequent item sets and give high compression ratios. Clearly, the MDL principle indicates that these small sets characterise the database. But, how characteristic are they? That is, do these small sets differentiate between different databases? In this paper we investigate this problem using classification. 
The small set of item sets (well, actually the code table they come from) compresses the original database well. Of course, this compression scheme can be used for all possible transactions. If the code compresses such an arbitrary transaction well, it "belongs" to the database.

This observation suggests a classification algorithm. Split the training database according to class and remove the items indicating the class from all transactions. Then, compute a code table for each of these databases. The set of code tables so derived form a classifier: a transaction $t$ gets assigned to the class $C$ whose code table compresses $t$ best.

The accuracy of this classifier is an independent characterisation of the quality of the small set of item sets our compression based mechanism picks from the huge set available. The experiments of this paper show that this classifier performs on par with well-known rule-induction and association-rule based methods. In other words, compression picks those sets that capture the data characteristics: the patterns that matter.

\section{Compression}

In this section we give a brief description of our compression based technique to filter out a small set of descriptive item sets from a vast amount of (frequent) item sets; a full description can be found in [11]. To make referring to our compression method easier, we will name it Krimp from now on (which is Dutch for "to shrink").

The first essential element of Krimp is a code table. Such a code table has item sets on the left-hand side and codes for these item sets on its right-hand side. The item sets in the code table are ordered descending on 1) item set length and 2) support. The actual codes on the right-hand side are of no importance, but their lengths are.

A transaction $t$ is encoded by Krimp by searching for the first item set $c$ in the lefthand side of the code table for which $c \subseteq t$. The code for $c$ becomes part of the encoding of $t$. If $t \backslash c \neq \varnothing$, the algorithm continues to encode $t \backslash c$. Since we insist that each coding table contains at least all singleton item sets, this algorithm gives a unique encoding to each (possible) transaction. The set of item sets used to encode a transaction is called its cover. Note that the coding algorithm implies that a cover consists of nonoverlapping item sets.

The length of the code of an item in a code table $C T$ depends on the database we want to compress; the more often a code is used, the shorter it should be. To compute this code length, we code each transaction in the database $d b$. The frequency of an item set $c \in C T$ is the number of transactions $t \in d b$ which have $c$ in their cover.

The relative frequency of $c \in C T$ is the probability that $c$ is used to encode an arbitrary $t \in d b$. For optimal compression of $d b$, the higher $\mathrm{P}(c)$, the shorter its code should be. In fact, we have the optimal code length [6] for $c$ as $-\log (\mathrm{P}(c))$.

The length of the encoding of a transaction is now simply the sum of the code lengths of the item sets in its cover. The size of the encoded database is the sum of the sizes of the encoded transactions. For the code table size, we only count those item sets that have a non-zero frequency. The size of the right-hand side column is obvious; it is simply the sum of all the different code lengths. For the left-hand side column, note that the simplest code table possibly consists of only the singleton item 
sets. This is the standard encoding which we use to compute the size of the item sets in the left-hand side column.

In [11] we defined the optimal set of item sets as that one whose associated code table minimizes the sum of the code table and encoded database sizes. The compression algorithm starts with a valid code table (generally only the collection of singletons) and a sorted list of candidates. These candidates are sorted descending on 1) support and 2) set length. Each candidate is considered by inserting it at the right position in $C T$ and calculating the new compressed size. A candidate is kept in the code table iff the resulting total size is smaller than it was before (Naïve-Compression).

The default Krimp algorithm, Compress-and-Prune, considers each existing code table element for pruning when a new candidate has been added: when an existing element does not contribute to compression it is permanently pruned. For more details, please see [11].

\section{Classification}

As usual in data mining, we assume that our database of transactions is an i.i.d. sample from some underlying data distribution. The result of any data mining algorithm is only useful if it reflects structure in the underlying distribution rather than spurious structure in the sample. Translated to Krimp, this means that we expect Krimp to compress an arbitrary transaction sampled from the underlying distribution well.

To make this more precise, assume that our code table $C T$ has no zero-frequency item sets. Let $t$ be an arbitrary transaction over the items $\mathcal{I}$, then:

$$
\begin{aligned}
l_{C T}(t) & =\sum_{c \in \operatorname{cover}(t)} l_{C T}(c)=\sum_{c \in \operatorname{cover}(t)}-\log (\mathrm{P}(c \mid d b)) \\
& =-\log \left(\prod_{c \in \operatorname{cover}(t)} \mathrm{P}(c \mid d b)\right)=-\log (\mathrm{P}(t \mid d b))
\end{aligned}
$$

The last equation is a Naïve Bayes [4] like assumption: we assume that the item sets that cover a transaction are independent. Clearly, this is overly optimistic as item sets in a cover are not allowed to overlap! However, Naïve Bayes is known to perform well, even if the independence assumption is violated. Therefore, we ignore this violation for the moment.

Now, assume that we have two databases $d b_{1}$ and $d b_{2}$ generated from two different underlying distributions. We apply Krimp to both databases and get two code tables, $C T_{1}$ and $C T_{2}$. We are given a new transaction $t$ that is generated under either the distribution for $d b_{1}$ or the one for $d b_{2}$, but we are not told which one. How do we decide to which distribution $t$ belongs? Under the Naïve Bayes assumption, we have:

$$
l_{C T_{1}}(t)<l_{C T_{2}}(t) \rightarrow \mathrm{P}\left(t \mid d b_{1}\right)>\mathrm{P}\left(t \mid d b_{2}\right)
$$

Hence, the Bayes optimal choice is to assign $t$ to the distribution that leads to the shortest code length. We already know that the result of Krimp is a small set of item sets that is optimal with regard to the MDL principle. The above observation suggests an independent way to assess the quality of this result: how well does it classify? 
The construction of the Krimp classifier works as follows:

1. Split the training database according to class

2. Remove the items that indicate the class from each transaction

3. Compress each of the databases, yielding a code table $C T_{i}$ for each class $C_{i}$

Then, to classify an unseen transaction $t$ :

1. Compute $l_{C T i}(t)$ for all classes $C_{i}$

2. Assign $t$ to the class that minimizes $l_{C T i}(t)$

Note that we do a Laplace correction during classification. That is, all usage frequencies are increased by one, ensuring valid code lengths for zero frequency sets. We generate code tables at fixed support intervals report-sup during compression, and use these for classification. To pair the code tables for classification we use two methods: absolute and relative. In absolute pairing, the code tables that have been generated at the same support levels are matched. Relative pairing matches code tables of the same relative support between $100 \%$ and $1 \%$ of max-sup (per class).

\section{Advanced Classifiers}

Many algorithms that can be used for classification have been proposed, many of which fall into either the class of rule-induction-based or that of association-rulebased methods. Because we use classification as a quality measure for the patterns that Krimp picks, we will compare our results with those obtained by some of the best existing classifiers. Comparison can be done with rule-induction-based methods such as C4.5 [9], FOIL [10] and CPAR [14], but we are more interested in an in-depth comparison with association-rule-based algorithms like iCAEP [16], HARMONY [12], CBA [7] and LB [8]. We believe this comparison is more interesting because these methods also use a collection of item sets for classification. Because we argued that our method is strongly linked to the principle of Naïve Bayes (NB) [4] its imperative we also compare to this method. Because these methods were devised with the goal of classification in mind, we would expect them to outperform the Krimp classifier. The goal of Krimp is extracting a small set of interesting patterns.

\section{Experiments}

In order to assess the quality of Krimp's data distribution approximation, we tested on a plethora of UCI databases and compared accuracies to those obtained by a large range of existing classification algorithms. As the algorithm currently only deals with item sets, we used discretised versions [3,5] of the databases.

All experimental results were obtained using 10-fold cross-validation. We report the min-sup thresholds we used for each dataset. We compare to scores taken from the publications in which the respective classifiers were described $[8,12,16]$. These all used the same discretised datasets. The missing scores for Naïve Bayes and C4.5 have been acquired using Weka [13]. 
Although virtually any collection of item sets could be considered as candidates, our focus has been on using all frequent and closed frequent item sets. Figure 1 shows that using either all or closed frequent item sets and NaiiveCompression or Compress-and-Prune hardly influences classification. Because the reduction of the number of item sets is largest with all frequent item sets and on-the-fly pruning [11], we use this combination in all experiments presented in the rest of this section.

Figure 2 shows that better compression generally leads to better classification. This is not always the case though:

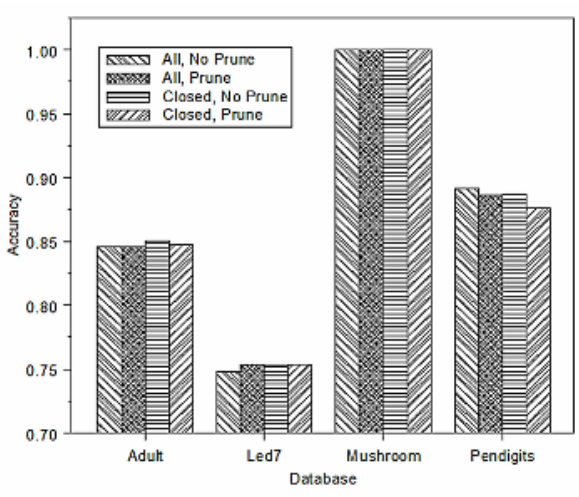

Fig. 1. Accuracies for different candidate sets (all/closed frequent item sets) and pruning enabled/disabled, for 4 datasets for the mushroom dataset, a drop in total compressed size always results in a change in accuracy, but this change is not always positive. The drops in accuracy could have several causes, the most likely being that item sets characteristic for both classes are added to the code tables at that support level. This would make the encoded probability distributions look more alike and make discrimination more difficult. The longer candidates with lower support make an important difference between the classes though, as a $100 \%$ classification score is obtained later on.

In case of the mushroom dataset, code tables for the two classes are paired absolute, e.g. having the same minimum support. This is not possible for the anneal dataset, as it consists of 5 classes that have a very skewed a priori distribution. However, using relative code table pairing, competitive scores can be obtained for anneal. The
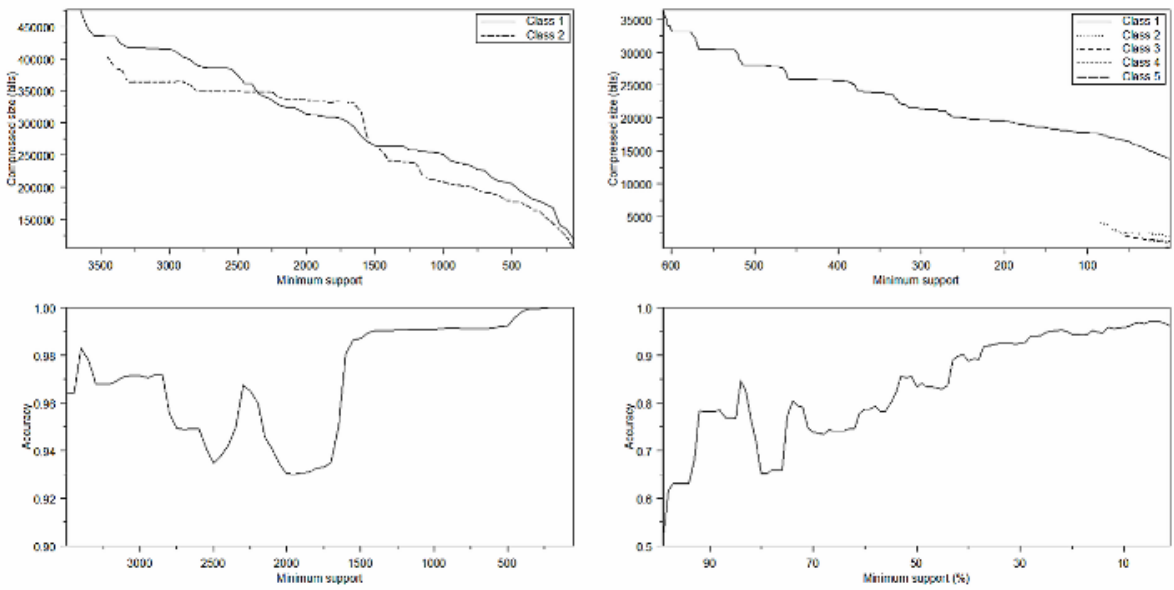

Fig. 2. Minimum support vs total compressed size per class and accuracy for mushroom (absolute pairing, left) and anneal (relative pairing, right) 
Table 1. Statistics on 8 UCI datasets using Compress-and-Prune with all frequent item sets as candidates. Numbers of candidates, resulting code table sizes $|\mathrm{CT}|$ (excluding singleton sets) and compression ratios at given min-sups are summed for all classes and 10-fold cross validated. The best 10-fold cross validated accuracies are given (not always belonging to min-sup).

\begin{tabular}{|c|c|c|c|c|c|c|c|c|}
\hline Dataset & \#rows & $\mathbf{I C l}$ & $|\mathcal{I}|$ & \#candidates & $|\mathrm{CT}|$ & $\begin{array}{c}\text { Compr } \\
\text { ratio }\end{array}$ & Acc \% & $\begin{array}{l}\min \\
\text { sup } \\
\end{array}$ \\
\hline Adult & 48842 & 2 & 97 & 1679483 & 994 & 3.40 & 84.6 & 50 \\
\hline Anneal & 898 & 6 & 71 & 2117941 & 110 & 2.47 & 97.0 & 1 \\
\hline Ionosphere & 351 & 2 & 157 & 42908227 & 107 & 1.47 & 90.6 & 35 \\
\hline Led7 & 3200 & 10 & 24 & 12815 & 720 & 3.49 & 75.3 & 1 \\
\hline Mushroom & 8124 & 2 & 119 & 92163948 & 391 & 3.94 & 100.0 & 50 \\
\hline Pendigits & 10992 & 10 & 86 & 255838056 & 1996 & 2.19 & 88.6 & 1 \\
\hline Waveform & 5000 & 3 & 101 & 942271 & 741 & 1.71 & 77.2 & 100 \\
\hline Wine & 178 & 3 & 68 & 1049213 & 167 & 1.26 & 97.7 & 1 \\
\hline
\end{tabular}

accuracy graph emphasizes that better compression doesn't always result in better classification: the highest accuracy is achieved shortly before min-sup is reached. This might be caused by slight overfitting.

In Table 1 we provide an overview of the achieved compression together with classification scores of Krimp on a variety of UCI datasets. The numbers clearly indicate that Krimp copes with a wide range of datasets and always strongly reduces the number of candidates. The reduction of the number of item sets is enormous, up to 5 orders of magnitude (in case of PenDigits, the beastly amount of 255 million item sets is cut back to only 1996!). Looking at the min-sups, only waveform seems like a strange outlier: because of its characteristics and density, compressing is computationally very intensive, despite the seemingly small numbers of rows and candidates.

As compression proceeds and classification improves, average encoded transaction lengths also change. In general, the difference between the length of the correctly (incorrectly) winning class and that of the losing class increases (decreases). This means that this difference could be used as uncertainty factor: a larger difference results in a higher certainty of assigning the correct class. (Not shown due to space limitations.)

The comparison of classification accuracies in Table 2 shows that the Krimp code tables are of such high quality they can compete with many methods specifically built for classification. Our elegant though make-shift MDL based classification extension

Table 2. Accuracy scores on 10 UCI datasets, 10-fold cross validated. Scores taken from [16], missing scores for Naive Bayes and C4.5 obtained using Weka [13].

\begin{tabular}{|l|r|r|r|c|c|r|r|r|r|}
\hline Dataset & \#cl & \multicolumn{1}{|c|}{ \#rows } & iCAEP & NB & LB & CBA & C4.5 & Krimp & $\begin{array}{c}\text { min } \\
\text { sup }\end{array}$ \\
\hline Adult & 2 & 48842 & 80.9 & 82.7 & 85.1 & 75.2 & $\mathbf{8 5 . 5}$ & 84.6 & 50 \\
\hline Anneal & 6 & 898 & 95.1 & 86.3 & & $\mathbf{9 8 . 1}$ & 90.4 & 97.0 & 1 \\
\hline Breast (Wisc) & 2 & 699 & $\mathbf{9 7 . 4}$ & 96.0 & 96.9 & 95.3 & 85.4 & 94.1 & 1 \\
\hline Iono & 2 & 351 & 90.6 & 82.6 & & $\mathbf{9 2 . 1}$ & 91.5 & 90.6 & 35 \\
\hline Iris & 3 & 150 & 93.3 & $\mathbf{9 6 . 0}$ & & 92.9 & 84.7 & $\mathbf{9 6 . 0}$ & 1 \\
\hline Mushroom & 2 & 8124 & 99.8 & 95.8 & & & $\mathbf{1 0 0 . 0}$ & $\mathbf{1 0 0 . 0}$ & 20 \\
\hline Pima & 2 & 768 & 72.3 & 74.7 & $\mathbf{7 5 . 8}$ & 73.1 & 72.5 & 75.0 & 1 \\
\hline Tic-tac-toe & 2 & 958 & 92.6 & 69.6 & & $\mathbf{1 0 0}$ & 84.6 & 87.1 & 1 \\
\hline Waveform & 3 & 5000 & 81.7 & 80.0 & 79.4 & 75.3 & 75.1 & 77.2 & 100 \\
\hline Wine & 3 & 178 & $\mathbf{9 8 . 9}$ & 96.6 & & 91.6 & 93.8 & 97.7 & 1 \\
\hline
\end{tabular}


Table 3. Accuracy scores on 8 large UCI databases, 10-fold cross validated. Scores taken from [12]. Min-sup for Harmony set to 50.

\begin{tabular}{|l|r|r|r|r|r|r|r|r|}
\hline Dataset & \#cl & \#rows & FOIL & CPAR & SVM & $\begin{array}{c}\text { Har- } \\
\text { mony }\end{array}$ & Krimp & $\begin{array}{c}\text { min } \\
\text { sup }\end{array}$ \\
\hline Adult & 2 & 48842 & 82.5 & 76.7 & 84.2 & 81.9 & 84.6 & 50 \\
\hline Led7 & 10 & 3200 & 62.3 & 71.2 & 73.8 & 74.6 & $\mathbf{7 5 . 3}$ & 1 \\
\hline LetterRecog & 26 & 20000 & 57.5 & 59.9 & 67.8 & $\mathbf{7 6 . 8}$ & 68.1 & 50 \\
\hline Mushroom & 2 & 8124 & 99.5 & 98.8 & 99.7 & 99.9 & $\mathbf{1 0 0 . 0}$ & 20 \\
\hline Nursery & 5 & 12960 & 91.3 & 78.5 & 91.4 & $\mathbf{9 2 . 8}$ & 92.4 & 1 \\
\hline PageBlocks & 5 & 5473 & 91.6 & 76.2 & 91.2 & 91.6 & $\mathbf{9 6 . 6}$ & 1 \\
\hline PenDigits & 10 & 10992 & 88.0 & 83.0 & 93.2 & $\mathbf{9 6 . 2}$ & 88.6 & 1 \\
\hline Waveform & 3 & 5000 & 75.6 & 75.4 & $\mathbf{8 3 . 2}$ & 80.5 & 77.2 & 100 \\
\hline
\end{tabular}

is always at the head of the pack and delivers two wins. Note that on average Krimp performs better than Naïve-Bayes, although it is based on the same assumption. Unfortunately no further results for LB and CBA were available.

When comparing on some of the largest UCI datasets in Table 3, Krimp proves to do exceptionally well. The bigger datasets allow MDL to better work its magic, making a good selection of which sets to include in its code tables. Overall, Krimp performs very well, as it is always close to the best scores and puts down the best score a number of times.

\section{Discussion}

Outstanding classification was not the ultimate goal of the experiments presented in the previous section, as explained before. We are very content as our explicit intention was to verify that our method is very capable at representing data distributions with only few item sets. The results of the experiments clearly verify this hypothesis.

Although Krimp has not been designed for classification and no attempts have been made to enhance the differences between code tables for different classes, the results show that it performs well compared to the best known classifiers. As the mentioned association-rule-based classifiers also select item sets that characterise the classes, it is interesting to compare our method with those in a qualitative way.

The selection method iCAEP uses is far less effective than MDL: the amount of Emergent Patterns may grow enormously and a lot of item sets may be required in the end. Also, Krimp is independent of the base distribution, iCAEP is not. Large Bayes does succeed in selecting only a small set of item sets that is used to determine a class-based probability distribution, but it uses an interestingness measure that requires a parameter that needs to be chosen manually.

HARMONY selects at most one rule per transaction in the training database. Although it is likely that equal rules are selected and therefore merged, the final set of rules can still be quite large. In Krimp, an item set is only used if it helps to compress the whole training database; we therefore believe that HARMONY is more prone to noise and overfitting than our method and the rules do not represent the data as well.

The elegancy of the classifier based on Krimp lies in 1) the use of only MDL for building and applying the classifier, 2) the small amount of item sets required and 3) the natural way it deals with multi-class problems and skewed class distributions. 


\section{Conclusion}

Krimp picks the item sets that matter. From staggering amounts it selects only handfuls of item sets that not only attain high compression ratios, but can compete with today's cutting edge classifiers as well. We therefore conclude that Krimp is well suited for capturing the characteristics of the data.

As an independent measure of the quality of the selected item sets we used classification. The training data is compressed per class, the resulting code tables are used to encode new test instances. Following Bayes optimal choice, the class with the code table assigning the shortest code has the highest probability of being the correct data distribution and is therefore chosen winner. Classification accuracies achieved are on par with the best known classifiers.

Not only is the selected collection of item sets of high quality, the reduction of the number of candidates is huge, generally many orders of magnitude. Krimp thus proves to be a generic method that finds small sets of patterns that encapsulate the probability distribution of the data well.

\section{References}

1. Agrawal, R., Imielinksi, T. \& Swami, A. Mining association rules between sets of items in large databases. In Proc. ACM SIGMOD conference, 207-216 (1993).

2. Bayardo, R. Efficiently mining long patterns from databases. In Proc. ACM SIGMOD conference, 85-93 (1998).

3. Coenen, F. The LUCS-KDD Discretised/normalized ARM and CARM Data Library. In www.csc.liv.ac.uk/ frans/KDD/Software/LUCS-KDD-DN/DataSets/dataSets.html.

4. Duda, R. \& Hart, P. Pattern Classification and Scene Analysis, John Wiley \& Sons, New York (1973).

5. Goethals, B. et al. FIMI website. In fimi.cs.helsinki.fi

6. Grünwald, P.D. Minimum description length tutorial. In Grünwald, P.D., Myung, I.J. \& Pitt, M.A., editors, Advances in Minimum Description Length. MIT Press (2005).

7. Liu, B., Hsu, W. \& Ma, Y. Integrating Classification and Association Rule Mining. In Proc. KDD-98, New York (1998).

8. Meretakis, D. \& Wüthrich, B. Extending Naïve Bayes Classifiers Using Long Itemsets. In Proc. of the Conference on Knowledge Discovery and Data Mining (1999).

9. Quinlan, J.R. C4.5: Programs for Machine Learning, Morgan Kaufmann (1993).

10. Quinlan, J.R. FOIL: A Midterm Report. In Proc. ECML '93 (1993).

11. Siebes, A.P.J.M., Vreeken, J. \& Van Leeuwen, M. Item Sets That Compress. In Proc. of the ACM SIAM Conference on Data Mining 393-404 (2006).

12. Wang, J. \& Karypis, G. HARMONY: Efficiently Mining the Best Rules for Classification. In Proc. of the ACM SIAM Conference on Data Mining (2005).

13. Witten, I.H. \& Frank, E. Data Mining: Practical machine learning tools and techniques, $2^{\text {nd }}$ edition, Morgan Kaufmann, San Francisco (2005).

14. Yin, X. \& Han, J. CPAR: Classification based on Predictive Association Rules. In Proc. SDM '03 (2003).

15. Zaki, M.J. \& Orihara, M. Theoretical foundations of association rules. In Proc. ACM SIGMOD workshop on research issues in KDD (1998).

16. Zhang, X., Guozhu, D. \& Ramamohanarao, K. Information-based Classification by Aggregating Emerging Patterns. In Proc. IDEAL (2000). 